\title{
Temperature Effect on Ferroelectric Polarization Switching in Poly(vinylidene fluoride)
}

\author{
Kazumi Matsushige, Satoshi Imada, and Tetuo TAKemura \\ Department of Applied Science, Faculty of Engineering, Kyushu University, \\ Hakozaki, Higashi-ku, Fukuoka 812, Japan.
}

(Received November 20, 1980)

\begin{abstract}
Ferroelectric polarization switching experiments were carried out on Form I crystal of poly(vinylidene fluoride) over a temperature range from $-76^{\circ} \mathrm{C}$ to $125^{\circ} \mathrm{C}$ and at applied electric fields $E$ up to $200 \mathrm{MV} \mathrm{m}^{-1}$. At $25^{\circ} \mathrm{C}$, notable polarization switching currents which decayed within a few $\mathrm{ms}$ were observed at $E \geqq 75 \mathrm{MV} \mathrm{m}^{-1}$. These currents became more intensive with increasing applied electric field. The maximum switching current density $J_{\max }$ and the maximum switching time $\tau_{\max }$ could be expressed approximately by $J_{\max }\left(A \mathrm{~m}^{-2}\right)=0.72(E-75)$ and $\tau_{\max }^{-1}$ $\left(\mathrm{s}^{-1}\right)=37(E-75)$, respectively, as also in the case with common ferroelectric crystals such as $\mathrm{BaTiO}_{3}$ and Rochelle salts. The polarization switching behavior changed remarkably with temperature and could be separated into three parts at about $-44^{\circ} \mathrm{C}$ and $60^{\circ} \mathrm{C}$. These temperatures coincide well with the reported $\beta$ (glass transition) and $\alpha$ (crystalline) relaxation temperatures, respectively. This fact indicates that the polarization switching phenomenon in poly(vinylidene fluoride) has close correlation with molecular chain motions. Finally, molecular motions responsible for the polarization switching in the three temperature regions are discussed.
\end{abstract}

KEY WORDS Poly(vinylidene fluoride) / Ferroelectric Polarization

Switching / Temperature Effect / Molecular Motions / Dipole Orientation /

Electric-Field Dependence /

Recent studies have revealed that Form I crystal of poly(vinylidene fluoride) (PVDF) has ferroelectric properties. ${ }^{1-10}$ From the phenomenological view point, the hysteresis characteristics in electric displacement, ${ }^{3}$ piezoelectric constant, ${ }^{3,4}$ and pyroelectric constant ${ }^{5}$ against the cyclic change of applied electric field were investigated. X-ray diffraction ${ }^{6,7}$ and infrared ${ }^{8-10}$ experiments demonstrated that the orientation of $\mathrm{CF}_{2}$ dipoles occurs by the application of high electric field, suggesting that this ferroelectric dipole orientation mainly contributes to a remnant polarization in PVDF. However, the details of the polarization reversal mechanism including the questions as to how large the polarization reversal units are and how fast the units switch have not as yet been clarified. The difficulty in answering the first question arises mainly from the situation that the domain structure reported for common ferroelectric crystals such as $\mathrm{BaTiO}_{3}$ and Rochelle salts has not been found for PVDF. In fact, even its existence is not definite for polymeric substances.
The second question can be answered if suitable experimental methods are employed. Since the electric displacement $D$ versus field $E$ hysteresis loops can be observed with $50 \mathrm{~Hz}$ electric field and the polarization switching should occur within a quarter of one cycle, the switching time is considered to be less than $5 \mathrm{~ms}$. Therefore, some special methods other than usual X-ray or infrared techniques should be employed in order to follow such a quick transient phenomenon.

In this study, polarization switching experiments using a step-wise application of voltage and highspeed digital memory scope were performed over wide ranges of temperature and applied electric field for Form I crystals of PVDF, and the correlation between the polarization reversal phenomenon and the molecular motions was investigated. Although the switching experiments on PVDF have been reported by Buchman, ${ }^{11}$ Okada and Toda, ${ }^{12}$ the experiments carried out by these authors are very limited in the range of temperature and amplitude 
(a)

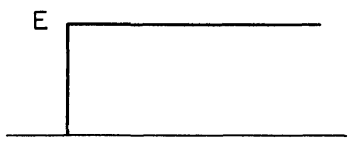

(b)

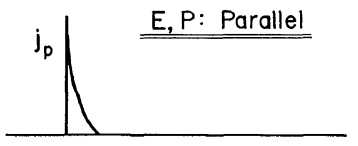

(c)

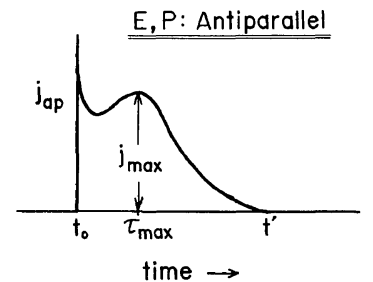

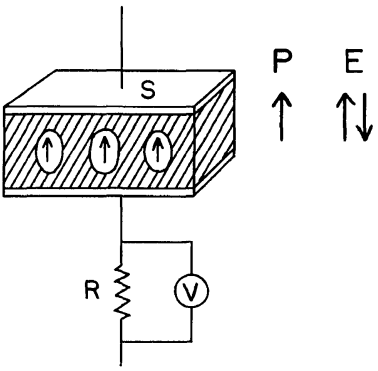

Switching Current Density $j=\frac{V}{R S}$

Polarization Change

$$
Q=\int_{t_{0}}^{t^{\prime}}\left(j_{a p}-j_{p}\right) d t
$$

Figure 1. Schematic presentation of the switching current technique: (a) applied step-wise electric field, (b) switching current profile when the directions of the applied electric field and of the dipoles existing in the sample are parallel, and (c) that when these directions are antiparallel to each other.

of applied electric field and did not cover the region where the ferroelectric properties of PVDF become apparent.

\section{EXPERIMENTAL}

\section{Material}

An original $50 \mu \mathrm{m}$ thick PVDF film, supplied by Kureha Chemical Industry Corp., was uniaxially stretched up to about five times its original length at $75^{\circ} \mathrm{C}$ and then heat-treated with its ends fixed at $130^{\circ} \mathrm{C}$ for one hour. The stretched film was confirmed by X-ray diffraction and infrared examinations to be an oriented Form I crystal containing a few percent of Form II crystals.

\section{Switching Current Measurement}

The principle of the switching current technique first employed by $\mathrm{Merz}^{13}$ for investigating $\mathrm{BaTiO}_{3}$ is schematically explained in Figure 1. When, as shown in Figure 1a, a high voltage is applied stepwise in the direction of molecular dipoles in the sample, a transient current $\left(j_{\mathrm{p}}\right)$ due only to a chargeup is observed (Figure 1b). On the other hand, when the directions are antiparallel, the large switching current $\left(j_{\mathrm{ap}}\right)$ arising from the polarization reversal of molecular dipoles can be detected (Figure 1c). Here, the switching current density $(j)$ and the polarization change $(Q)$ are defined by $j=V / R S$ and
$Q=\int_{t_{0}}^{t \prime}\left(j_{\mathrm{ap}}-j_{\mathrm{p}}\right) \mathrm{d} t$, where $V, R$, and $S$ are voltage, resistance, and electrode area, respectively. In order to eliminate the influence of the $j_{\mathrm{p}}$ component from the observed $j_{\text {ap }}$ value, the calculation of $j=j_{\text {ap }}-j_{\mathrm{p}}$ was made electrically, using the subtraction circuit shown in the next figure and the maximum switching time $\left(\tau_{\max }\right)$ and the maximum switching current density $\left(j_{\max }\right)$ were defined at the peak position in the $j\left(=j_{\mathrm{ap}}-j_{\mathrm{p}}\right)$ vs. time curves thus obtained. In order to achieve the saturation of the dipole orientation at the electric field $E$, the electric field $E$ was applied more than ten times to the sample in the same direction repeatedly, and then the measurement of $j_{\text {ap }}$ was performed by applying the first reversed electric field $E$ to the sample.

A schematic diagram of the switching experimental system is shown in Figure 2. High d.c. voltage steps were applied to the sample through a switching device. The device consisted of 18 pieces of reed relays (Oki Electric, RRF51A05P) connected in series and its $90 \%$ rise-up time was about $100 \mathrm{~ns}$. The measurements were performed with a digital memory scope (Iwatsu, DMS-6430) at a clock rate of $10 \mu$ s per WD and a data length of $1024 \mathrm{WD}$. The applied voltage was monitored through $\mathrm{CH} 1$ and the switching current, which was detected as the voltage change appearing at the ends of resistance $R_{4}$, was observed through $\mathrm{CH} 2$. The stored signals were displayed on an oscilloscope and recorded on a 


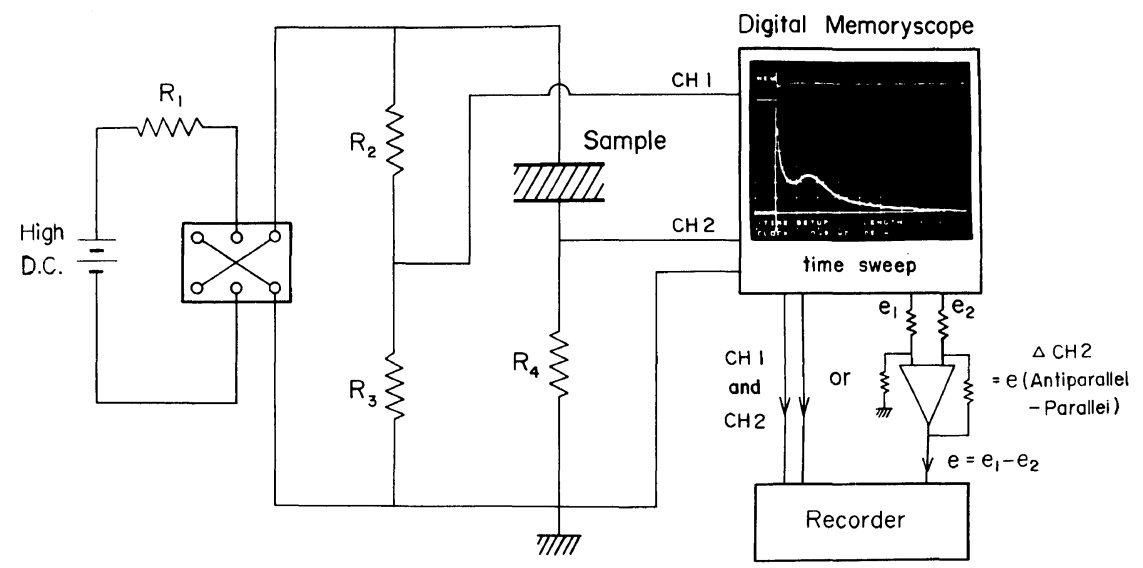

Figure 2. Schematic diagram of a switching experimental system.

chart paper directly or through a subtraction circuit.

The sample cell is shown in Figure 3. A film sample was sandwiched between a pair of brass anvils whose surfaces were polished very smoothly. The brass anvils were supported with a spring to obtain close contact between the sample and the brass anvils; also, the sample cell as a whole was immersed in a silicone oil bath in order to prevent a discharge phenomenon from taking place between the electrodes. Film samples were tested successfully without evaporated metal electrodes on the film surfaces. This gave us an advantage of saving the time for metal evaporations which sometimes cause dimensional shrinkages and structural changes to stretched film samples. Since there existed slight roughness of a few $\mu \mathrm{m}$ on the sample surfaces, this might cause some discrepancy between the apparent and real amplitudes of the applied electric field. The temperature was controlled by a heater and/or liquid $\mathrm{N}_{2}$, and monitored with a chromel-alumel thermocouple.

\section{RESULTS AND DISCUSSION}

\section{Electric-Field Dependence}

In order to study the effect of the amplitude of an applied electric field on switching current behavior, the time dependence of the switching current density ( $j-t$ curves) was measured at $25^{\circ} \mathrm{C}$ and at various applied electric fields, and the results are shown in Figure 4. When the electric field $\mathrm{E}$ was lower than about $50 \mathrm{MV} \mathrm{m}^{-1}$, the switching current

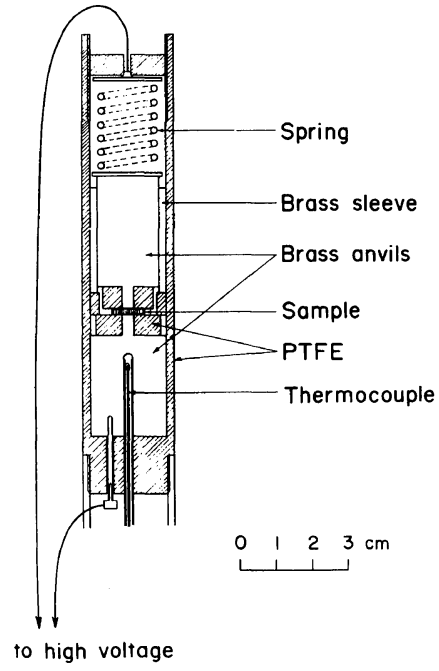

Figure 3. Sample cell for the switching experiment.

was scarcely observable, but notable switching currents were detected at $E$ values higher than about 75 $\mathrm{MV} \mathrm{m}{ }^{-1}$. With increasing $E$, the peaks of the $j-t$ curves became higher and their positions (indicated by arrows) shifted toward shorter time. From the observed $j-t$ curves, the electric-field dependences of three characteristic quantities $Q, \tau_{\max }$, and $j_{\max }$ were obtained as shown in Figure 5. With increasing $E, Q$ increases linearly and then gradually shows a saturation tendency. The $Q$ value observed at $E=200$ $\mathrm{MV} \mathrm{m} \mathrm{m}^{-1}$ was $103 \mathrm{mC} \mathrm{m}^{-2}$, which suggests that about $45 \%$ of $\mathrm{CF}_{2}$ molecular dipoles in the sample was oriented toward the applied electric field based on the following assumptions: a residual polari- 
zation $\left(P_{\mathrm{r}}=Q / 2\right)$ in PVDF sample exists only in the crystalline phase, a spontaneous polarization of PVDF Form I crystallites which are dispersed in an amorphous matrix is about $230 \mathrm{mC} \mathrm{m}^{-2},{ }^{14}$ and the crystallinity of the test sample is $50 \%$. On the other hand, $j_{\max }\left(A \mathrm{~m}^{-2}\right)$ increases linearly with increasing $E$, as can be expressed by $j_{\max }=0.72(E-75)$, where the unit of $E$ is in $\mathrm{MV} \mathrm{m} \mathrm{m}^{-1}$, except for the toe region. Although $\tau_{\max }$ decreases non-linearly with

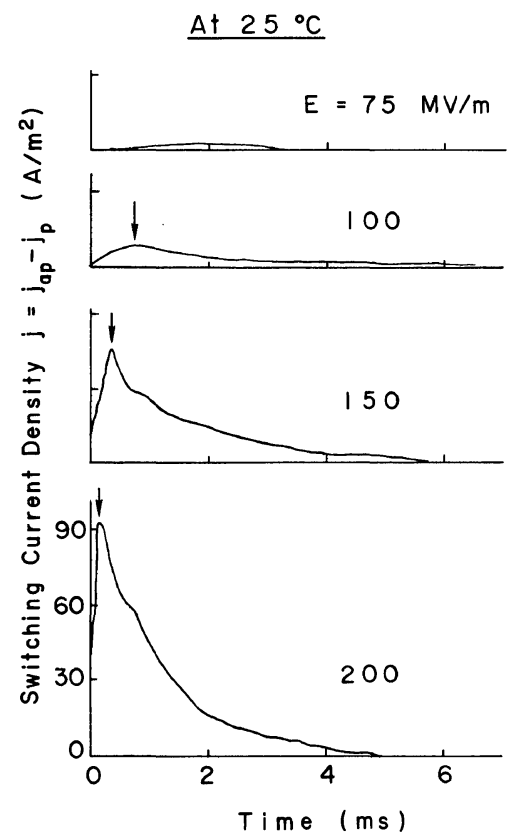

Figure 4. Switching current profiles observed at $25^{\circ} \mathrm{C}$ and at different electric fields. Arrows indicate peak positions. increasing $E$, the $\tau_{\max }^{-1}\left(\mathrm{~s}^{-1}\right)$ can be fitted by a linear relation $\tau_{\max }^{-1}=37(E-75)$.

These experimental relations suggest that the molecular motions involved in the polarization reversal are activated by application of an electric field whose amplitude is above some critical value $E_{\mathrm{c}}$ and that the amount of the polarization reversal units and their movements are both enhanced by the driving force $E-E_{\mathrm{c}}$. The $E_{\mathrm{c}}$ value observed here is apparently higher than the $50 \mathrm{MV} \mathrm{m}^{-1}$ reported by Furukawa et al. ${ }^{15}$ This discrepancy is thought to originate from differences in the measuring methods. It is well known that the polarization reversal phenomenon is time-dependent and the $E_{\mathrm{c}}$ value depends on the time rate (frequency) of the applied electric field. ${ }^{16}$ The frequency employed by Furukawa et al. was $1 / 300 \mathrm{~Hz}$, which is considerably slower than the one used in our switching experiment. Another difference is in the sample preparations. In this study, the film samples were tested without evaporated metal electrodes, so that a slight clearance between the sample surfaces and brass anvil electrodes might have reduced the real amplitude of applied electric field. Both of these two differences are.considered to be responsible for the larger $E_{\mathrm{c}}$ value in our observation compared to the reported one.

It is noteworthy that the electric field dependences of $j_{\max }$ and $\tau_{\max }^{-1}$ observed here are quite similar to those reported for common ferroelectric crystals such as $\mathrm{BaTiO}_{3}{ }^{13}$ and Rochelle salts. ${ }^{17}$ The measured values of $j_{\max }, \tau_{\max }$, and $E_{\mathrm{c}}$ for PVDF, however, are about one hundredth, one thousandfold, and ten thousandfold the values for these

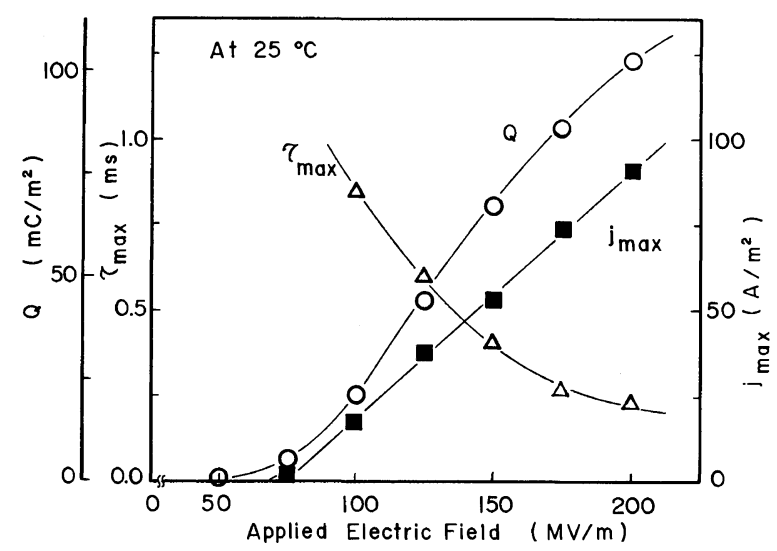

Figure 5. Applied electric field dependences of $Q, j_{\max }$, and $\tau_{\max }$ at $25^{\circ} \mathrm{C}$. 


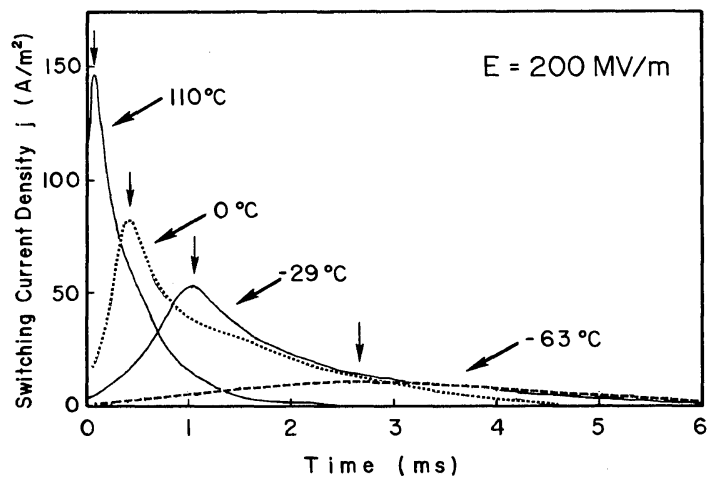

Figure 6. Switching current profiles observed at $E=200 \mathrm{MV} \mathrm{m}^{-1}$ and at different temperatures.

common ferroelectric crystals. This marked difference indicates that the polarization reversal in PVDF is extremely difficult and requires a driving energy greater than that in common ferroelectric crystals. This may be attributed to the fact that the polarization reversal in PVDF must involve the rotation of molecular chains or larger crystalline units and/or conformational changes of molecular chains, while that in common ferroelectric crystals can be achieved easily either. by movement of hydrogen atoms or by displacement of ionic atoms in crystalline lattices.

\section{Temperature Effect}

Switching experiments were carried out in the temperature range from $-76^{\circ} \mathrm{C}$ to $125^{\circ} \mathrm{C}$ in order to investigate the correlation between the polarization reversal phenomenon and molecular chain motions in PVDF. As shown in Figure 6, the switching current profiles at $E=200 \mathrm{MV} \mathrm{\textrm {m } ^ { - 1 }}$ change drastically with temperature. At $-63^{\circ} \mathrm{C}$, the profile is very broad and exhibits a peak at around $2.7 \mathrm{~ms}$. With increasing temperature, the peak positions shift toward shorter times; thus $1.1 \mathrm{~ms}$ at $-29^{\circ} \mathrm{C}, 0.4 \mathrm{~ms}$ at $0^{\circ} \mathrm{C}$, and $0.2 \mathrm{~ms}$ at $110^{\circ} \mathrm{C}$.

The switching current profiles at various temperatures had similar shapes, though different in the scales of current and time axes. Thus, it appeared that these profiles were 'affine' as reported for common ferroelectric crystals. ${ }^{18}$ However, a close examination indicates that they were not really 'affine' but rather composed of two components, a primary peak and a secondary peak indicated by the arrow in Figure 7. The secondary peak can be observed

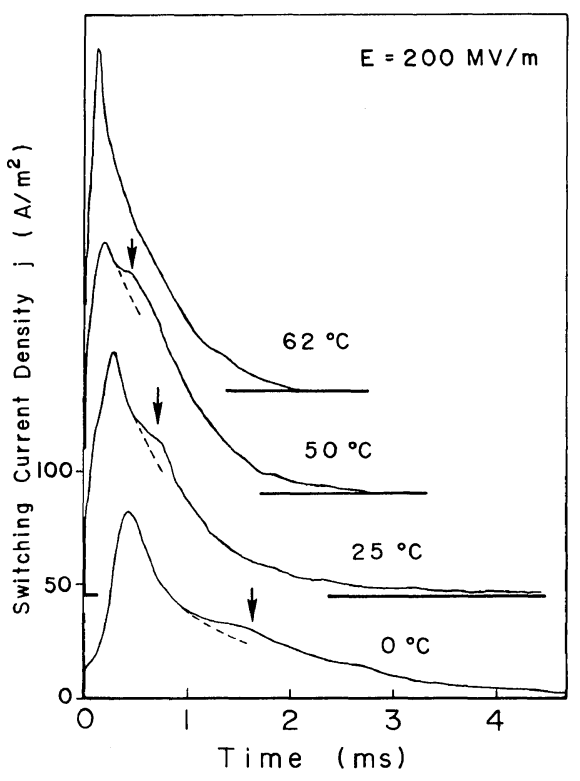

Figure 7. Switching current profiles observed at $E=200 \mathrm{MV} \mathrm{m}^{-1}$ and in the temperature range from $0^{\circ} \mathrm{C}$ to $62^{\circ} \mathrm{C}$. Arrows indicate the positions of the secondary peak.

only in the limited temperature range from $0^{\circ} \mathrm{C}$ to $50^{\circ} \mathrm{C}$. With increasing temperature, the position of the secondary peak shifts toward a shorter time much faster than the primary peak and the existence of secondary peak can hardly be detected at temperatures above $62^{\circ} \mathrm{C}$. Because of this overlapping of the secondary peak with the primary peak, the value of $j_{\max }$ is abnormally high at $62^{\circ} \mathrm{C}$.

Figure 8 shows the temperature dependences of $Q, \tau_{\max }, \tau_{\max }^{\mathrm{s}}$ (the maximum switching time of the 


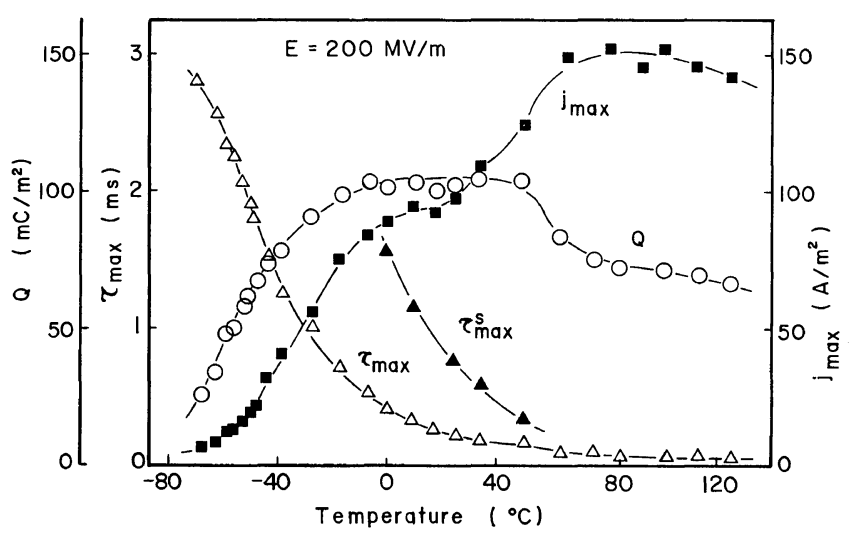

Figure 8. Temperature dependences of $Q, \tau_{\max }, \tau_{\max }^{\mathrm{s}}$, and $j_{\max }$. The data are taken from the switching current profiles observed at $E=200 \mathrm{MV} \mathrm{m}^{-1}$

secondary peak), and $j_{\max }$. The $Q$ values steeply increase around $-60^{\circ} \mathrm{C}$, then become almost constant and finally decrease gradually above $60^{\circ} \mathrm{C}$. This temperature dependence of $Q$ is quite similar to that of the piezoelectric constant reported by Tamura et al..$^{19}$ and Furukawa et al. ${ }^{20}$ suggesting that the piezoelectricity in PVDF has a close correlation with the remnant polarization. The $\tau_{\max }$ values decrease monotonously with increasing temperature and become less than one tenth the values at low temperatures. The $\tau_{\max }^{\mathrm{s}}$ values decrease similarly with increasing temperature but much faster than the $\tau_{\max }$ values. On the other hand, the $j_{\max }$ values show a temperature dependence similar to that of $Q$ except in the temperature range from $40^{\circ} \mathrm{C}$ to $60^{\circ} \mathrm{C}$. The substantial raise in the $j_{\max }$ value observed in this temperature range is considered due to the above-mentioned overlapping of the secondary and the primary peak.

Next, in order to perform thermodynamic analysis of the molecular chain motions associated with the polarization reversal phenomenon, the values of $\ln \tau_{\max }^{-1}$ were plotted against $1 / T(\mathrm{~K})$, as shown in Figure 9 . The data points are fitted by three straight lines with two breaking points at around $-44^{\circ} \mathrm{C}$ and $60^{\circ} \mathrm{C}$. It is notable that these two special temperatures correspond well to the reported $\beta$ and $\alpha$ relaxation temperatures of Form I crystal, ${ }^{21-24}$ respectively. The $\beta$ relaxation is related to microbrownian motions of amorphous chain segments, while the $\alpha$ relaxation is associated with the molecular motions in the interior of crystalline regions. The data of Figure 9 give apparent activation

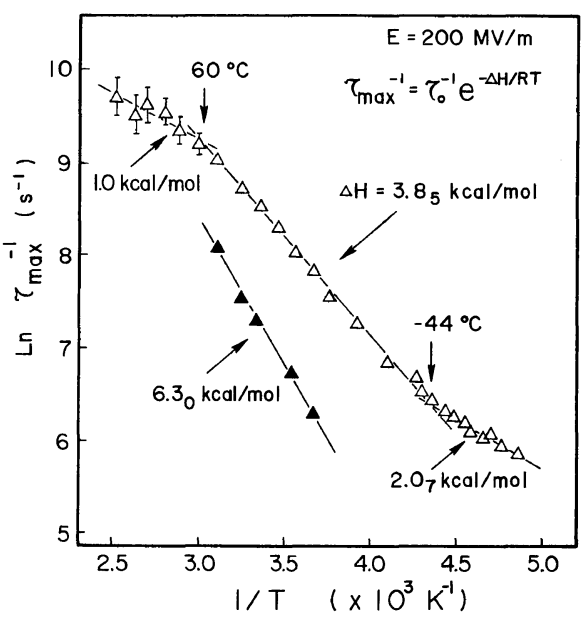

Figure 9. Activation plots of $\tau_{\max }$ and $\tau_{\max }^{\mathrm{s}}$.

energies of $1.0,3.8_{5}$, and $2.0_{7} \mathrm{kcal} \mathrm{mol}^{-1}$ for the three linear regions bounded by the two breaking points in the order of decreasing temperature. On the other hand, the data for the secondary peak shown in Figure 9 give a larger activation energy of $6.0_{3} \mathrm{kcal} \mathrm{mol}^{-1}$. To ascertain the validity of these activation energy values, it is necessary to study in detail the classification or identification of the molecular motions involved in the polarization reversal phenomenon and on the theoretical calculation for these molecular motions.

As described above, the temperature dependence of the polarization reversal phenomenon in PVDF can be separated into three stages. We may suggest molecular motions responsible for each stage as 
follows, on the basis of the experimental results. Stage I (the temperature range below $-44^{\circ} \mathrm{C}$ ): In this temperature region, only local molecular motions in the amorphous region are activated, and these may be capable of responding to the application of an electric field. Therefore, the activation energy for this stage is not very large and the extent of polarization reversal, i.e., the $Q$ value, should be small, as was actually observed. Stage II (the temperature range between $-44^{\circ} \mathrm{C}$ and $60^{\circ} \mathrm{C}$ ): Since the temperature in this stage is higher than the glass transition temperature but lower than the crystalline relaxation temperature, microbrownian motions of amorphous chain segments are activated. Also, the molecular chains in the crystallites which are dispersed in the amorphous region and bound by flexible tie molecules may become movable or rotatable by applying a high electric field. These molecular movements are considered responsible for the polarization reversal in this temperature region. As the temperature increases, the reversing speed and the amount of reversed molecular dipoles become faster and larger due to the temperatureenhanced molecular motions, resulting in a drastic decrease in the $\tau_{\max }$ value and an increase in the $Q$ value, respectively. On the other hand, the origin of the secondary peak is less clear. However, the fact that the secondary peak has a longer $\tau_{\max }^{\mathrm{s}}$ and a higher activation energy than the primary peak and cannot be distinguished from the primary peak above the crystalline relaxation temperature suggests that the molecular units responsible for the secondary peak are larger than those for the primary peak. Stage III (the temperature range above $60^{\circ} \mathrm{C}$ ): Above the crystalline relaxation temperature, various molecular motions including those in the interior of crystalline regions are thermally activated. Therefore, the dipole orientation can be achieved much more easily by the application of an electric field, resulting in a low activation energy and a very short switching time. On the other hand, the observation that the $Q$ value is lower in this temperature region is thought to be due to a situation such that even if a very high degree of dipole orientation were once achieved on application of an electric field, the thermally activated molecular motions would cause the decay of the oriented state in a short time and give rise to a lower residual polarization state.

In order to further clarify the molecular motions responsible for the polarization reversal phenomenon, we are carrying out the switching experiment at high pressure and measuring the compressibilities of crystalline and amorphous parts in PVDF. The results will be published soon.

Acknowledgement. This work was supported in part by a Grant-in Aid for Science Research from the Ministry of Education, Science and Culture.

\section{REFERENCES}

1. K. Nakamura and Y. Wada, J. Polym. Sci., A-2, 9, 161 (1971).

2. J. H. McFee, J. G. Bergman, Jr., and G. R. Crane, Ferroelectrics, 3, 305 (1971).

3. M. Tamura, K. Ogasawara, N. Ono, and S. Hagiwara, J. Appl. Phys., 45, 3768 (1974).

4. M. Oshiki and E. Fukada, J. Mater. Sci., 10, 1 (1975).

5. K. Ogasawara, K. Shiratori, and M. Tamura, Rep. Prog. Polym. Phys. Jpn., 19, 313 (1976).

6. G. T. Davis, J. E. McKinney, M. G. Broadhurst, and S. C. Roth, J. Appl. Phys., 49, 4998 (1978).

7. D. K. Das-Gupta and K. Doughty, J. Appl. Phys., 49, 4601 (1978).

8. P. D. Southgate, Appl. Phys. Lett., 28, 250 (1976).

9. M. Date and E. Fukada, Rep. Prog. Polym. Phys. Jpn., 20, 339 (1977).

10. D. Naegele and D. Y. Yoon, Appl. Phys. Lett., 33, 132 (1978).

11. P. Buchman, Ferroelectrics, 5, 39 (1973).

12. Y: Okada and M. Toda, "Proceedings of the 1st Meeting on Ferroelectric Materials and Applications, Kyoto," S. Marutake, Ed., Kyoto University Press, Kyoto, 1977, p 245.

13. W. J. Merz, J. Appl. Phys., 27, 938 (1956).

14. Y. Wada and R. Hayakawa, Rep. Prog. Polym. Phys. Jpn., 23, 417 (1980).

15. T. Furukawa, M. Date, and E. Fukada, J. Appl. Phys., 51, 1135 (1980).

16. C. F. Pulvari and W. Kuebler, J. Appl. Phys., 29, 1315 (1958).

17. H. H. Wieder, Phys. Rev., 110, 29 (1958).

18. J. C. 'Burfoot, "Ferroelectrics," D. Van Nostrand Company, Ltd. London, 1967, p 215.

19. M. Tamura, S. Hagiwara, S. Matsumoto, and N. Ono, J. Appl. Phys., 48, 513 (1977).

20. T. Furukawa, J. Aiba, and E. Fukada, J. Appl. Phys., 50, 3615 (1979).

21. N. Koizumi, S. Yano, and K. Tsunashima, J. Polym. Sci., B, 7, 59 (1969).

22. H. Kakutani, J. Polym. Sci., A-2, 8, 1177 (1970).

23. S. Yano, J. Polym. Sci., A-2, 8, 1057 (1970).

24. K. Nakagawa and Y. Ishida, J. Polym. Sci. Polym. Phys. Ed., 11. 1503 (1973). 